\title{
“Do Not Attempt Resuscitation" Draft Guidelines, Google Al, and Trends to Watch in 2020
}

In December 2019, the Indian Council of Medical Research (ICMR) has released a draft document to guide physicians on decisions not to provide cardiopulmonary resuscitation (CPR) to patients. The draft document narrates that the death is inevitable in certain situations at the terminal stage of certain diseases and providing CPR may only increase the suffering of such patients. The draft document recommends that physicians should provide complete information to patients or their family members about circumstances under which CPR would in the best judgment of physicians be considered medically inappropriate. It emphasizes the need to explain such circumstances to patients and family members, so they understand the implications of CPR in the context of the incurability of the disease and the nearly impossible opportunity for any meaningful survival.

The draft document issued by ICMR provides information such as an algorithm, with a flowchart guiding the physicians on how best to proceed, and in which circumstances CPR should be provided. It will guide treating physicians to take the decision whether or not to perform the CPR on the background of incurable disease where the patient's chances of survival are extremely low. The flowchart provided by ICMR is provided in Figure 1.

ICMR has invited feedback and comments on the Draft ICMR Position Paper on "Do Not Attempt Resuscitation (DNAR)" from relevant stakeholders. It is requested that comments/feedback pertaining to the same may be conveyed in the prescribed format by E-mail to Dr. Roli Mathur (icmr.bioethics@gmail.com).

The guidelines on such topics are very sensitive topic, as there will always be those who oppose the decision. Leaving an individual to pass away will cause distress and unhappiness for family and friends. However, the draft document does highlight the importance of providing detailed yet simply understood reasoning to the family of the affected individual through every step of the decision-making process.

Until now, there has been no formal classification or guidelines provided by any government body to establish an appropriate course of action in such a circumstance. This places physicians in a difficult situation in which neglecting to perform CPR can have terrible repercussions both to their career and, indeed, to their safety.

The question which will also be debated is priority when the patient requires CPR (especially in patients

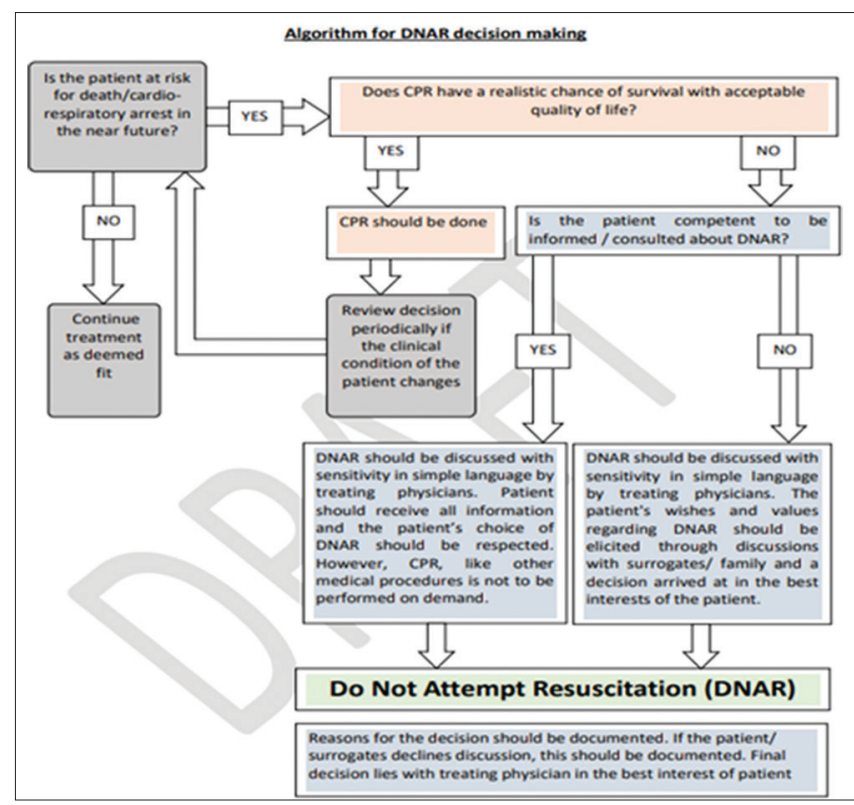

Figure 1: Algorithm for do not attempt resuscitation decision making (from draft document issued by the Indian Council of Medical Research)

who are brought to emergency department); whether the physician shall spend time in explaining patient's relatives about DNAR rather than actually providing CPR to the patient. The time spent at that time by the physician on DNAR may be looked differently leading arguments.

Considering the sheer volume of violence committed against physicians, many are in a situation where they may feel threatened in opting not to perform CPR. Although following guidelines on the practice will, of course, not alleviate the threat of violence in its entirety, it can, at the very least, help inform the family as to decisions made in terms of giving CPR.

It will be crucial to know interest taken by physicians in reporting comments on the draft document to ICMR and contents of finalized document once released by ICMR post addressing comments.

\section{Google AI Platform aids in Breast Cancer Screenings}

The year 2020 started with a big news in health-care system mainly from the perspective of positive implications of artificial intelligence (AI) on cancer detection. Google Health announced the results of a study which indicates AI could be more effective in spotting breast cancer. The findings, published in nature, indicate that Google's AI model spotted breast cancer in deidentified screening 
mammograms with greater accuracy, with fewer false positives and false negatives than experts.

Google's London-based AI subsidiary DeepMind worked with Cancer Research UK Imperial Centre, Northwestern University and Royal Surrey County Hospital to train and deploy the AI model. The UK test set consisted of screening mammograms that were collected between 2012 and 2015 from 25,856 women at two screening centers in England, where women are screened every 3 years. This was a random sample of $10 \%$ of all women with screening mammograms at these sites during this time period. The UK cohort resembled the broader screening population in age and disease characteristics. The test set from the USA, where women are screened every 1-2 years, consisted of screening mammograms that were collected between 2001 and 2018 from 3097 women at one academic medical center.

The study showed that the AI system could identify cancers with a similar level of accuracy to expert radiologists. At the same time, it reduced the number of false positive results by $5.7 \%$ in the American patients and $1.2 \%$ in the British patients. It also cut the number of false negatives, where tests are wrongly listed as normal, by $9.4 \%$ in the American group and $2.7 \%$ in the British group. These results show differences in how mammograms are read. In the USA, only one radiologist reads the results and the tests are done every 1-2 years. In Britain, the tests are done every 3 years, and each is read by two radiologists. When they disagree, a third radiologist reads it.

The study has some limitations. Most of the tests were done using the same type of imaging equipment, and the USA group had a lot of patients with confirmed breast cancers. The next challenge lies in getting regulatory approval which may take few years.

The use of machine learning technologies in breast cancer screening could have huge implications, as spotting and diagnosing breast cancer early remains a challenge with radiologists.

This is not the first time AI has shown big promise for better mammography detection. In 2016, researchers at Houston Methodist developed (Houston Methodist Research Institute, Houston, USA) an AI software which could improve readings to $99 \%$ accuracy by analyzing values from X-ray images and the text of clinical reports. In April 2019, CureMetrix (health-care technology company based in California, USA) has received the Food and Drug Administration (FDA) approval to sell its software "triage" service to screen mammograms for signs of cancer. The AI-based triage software for mammography named as cmTriage is a workflow optimization tool that enables a radiologist to customize, sort, and prioritize their mammography worklist based on cases that may need immediate attention. Rather than today's method of first in, first out for reading through a screening mammography worklist, radiologists can now use cmTriage to identify which cases are suspicious and focus on those first. They can also use cmTriage to optimize their clinical workflow by sending the suspicious cases to their most senior radiologists or reading the suspicious cases immediately before the patient has even left the clinic. This workflow optimization tool enables a radiologist to customize their mammography worklist based on cases that may need immediate attention.

The opportunities for clinical improvements using AI are broad and diverse, and the technology stands to continue making big advances across health care in 2020. For instance, AI technology based on a deep learning model has also shown promise of helping cardiologists to predict irregular heart rhythm, atrial fibrillation, before it develops.

The medical fraternity will be looking forward to future applications; there are some promising signs that the model could potentially increase the accuracy and efficiency of screening programs and reduce wait times and stress for patients. However, ensuring its reach to treating physicians post conduct of clinical trials and regulatory approvals will be critical in acceptance and use of such technologies.

\section{Trends to Watch Out for in 2020}

Direct-to-consumer drug delivery - set to become more common in 2020

The pharma companies in India are under lot of pricing pressure and expected to think innovative ways to reduce cost to patients. Choosing direct-to-consumer (DTC) may help pharma companies in reducing margins to be shared with distributors and effectively reduce the cost. DTC drug delivery firms could give pharma firms more direct access to consumers and their medication-related habits. Using DTC startups working in the drug delivery to consumer space would be a smart way for pharma.

Big pharma companies probably would not embark on acquisitions of such companies which provide DTC services for a revenue boost as most of these DTC companies are start-ups. Few names in the space of DTC such as Nurx, Hims and Hers, and capsule have all grabbed funding this year and consumers are becoming more and more convenience-driven, which will likely increase demand for fast and reliable drug delivery services.

\section{Emphasis on targeted therapies}

Several years ago, personalized medicine was an emerging concept rather than a concrete part of the health-care system. However, last year, $42 \%$ of newly approved drugs, agents, or therapeutic biologics were classified as personalized medicines.

Personalized medicine refers to therapies that are designed based on the genetic and molecular makeup of a patient. 
In principle, an individualized treatment should be more effective at treating a patient compared to a nonspecific drug.

With the advancement of genetic techniques and a greater understanding of disease pathology, the cancer drug development industry, in particular, has witnessed a major shift toward targeted therapies. In the last 3 years alone, the US FDA approved eight targeted therapies for acute myeloid leukemia.

\section{Rise in biosimilars and generics}

A biosimilar is highly similar to and has no clinically meaningful differences from an existing FDA-approved reference product, according to the FDA. In other words, biosimilars do not necessarily contain the same active ingredients as the reference product. In contrast, generic drugs do contain the same active ingredients as those of the brand name drugs. Biosimilars and generics are often cheaper than brand-name drugs, but are each regulated differently.

The industry has seen a rise in biosimilar and generic drugs as decades-old patents begin to expire. The Brazilian, Russian, Indian, and Chinese drug markets are each expected to spend about double the amount on generics compared to patented products.

\section{Reimagined clinical trials}

In 2020, sponsors can look forward to new innovations into the clinical trial space, including more virtual or hybrid trials, adaptive trials, and other designs that better meet patient needs. In fact, companies are recognizing the value of patient insights (and other stakeholders) for successful trial execution.

Trials are also becoming more complex than ever before. The amount of data that is available to sponsors comes as a blessing in disguise - more insights can lead to more effective treatment, yet the disparate sources of data present a significant challenge for accurate and timely analysis.
Sophisticated digitization tools promise to streamline clinical data management, reduce costs, and accelerate study completion times.

Financial support and sponsorship

Nil.

\section{Conflicts of interest}

There are no conflicts of interest.

\section{Ganesh H Divekar ${ }^{1}$, Padmaj S Kulkarni²}

${ }^{I}$ Clinical Operations and Medical Services, SIRO Clinpharm Pvt. Ltd., Thane, Maharashtra, India, ${ }^{2}$ Department of Medical Oncology, Deenanath Mangeshkar Hospital, Pune, Maharashtra, India

Address for correspondence: Dr. Ganesh H Divekar, $1^{\text {st }}$ Floor, Unit Nos. 3 and 4, Plot No. D-3, Road No. 16, Wagle Industrial Estate, Thane West, Thane, Maharashtra - 400 604, India. E-mail: ganesh.divekar@gmail.com

Submitted: 09-Feb-2020 Accepted in Revised Form: 12-Mar-2020

Published: 24-Apr-2020

This is an open access journal, and articles are distributed under the terms of the Creative Commons Attribution-NonCommercial-ShareAlike 4.0 License, which allows others to remix, tweak, and build upon the work non-commercially, as long as appropriate credit is given and the new creations are licensed under the identical terms.

\begin{tabular}{|l|l|}
\hline \multicolumn{2}{|c|}{ Access this article online } \\
\hline Quick Response Code: & Website: \\
& www.ijmpo.org \\
\cline { 2 - 2 } & DOI: \\
\hline
\end{tabular}

How to cite this article: Divekar GH, Kulkarni PS. "Do not attempt resuscitation" draft guidelines, Google Al, and trends to watch in 2020. Indian J Med Paediatr Oncol 2020;41:75-7. 